\title{
IMRT combined with concurrent chemotherapy plus adjuvant chemotherapy versus IMRT combined with concurrent chemotherapy alone in patients with nasopharyngeal carcinoma
}

\author{
Qiulu Zhong ${ }^{1}$, Xiaodong Zhu ${ }^{1}$, Ling Li ${ }^{1}$, Song Qu ${ }^{1}$, Zhongguo Liang ${ }^{1}$, Fanyan Zeng ${ }^{1}$ \\ and Xinbin Pan ${ }^{1}$ \\ ${ }^{1}$ Department of Radiation Oncology, The Affiliated Cancer Hospital of Guangxi Medical University, Cancer Institute of Guangxi \\ Zhuang Autonomous Region, Nanning, Guangxi, China \\ Correspondence to: Xiaodong Zhu, email: zhuxdonggxmu@126.com \\ Keywords: nasopharyngeal carcinoma; intensity-modulated radiotherapy; concurrent chemoradiotherapy; concurrent chemora- \\ diotherapy plus adjuvant chemotherapy \\ Received: August 15,2016 Accepted: December 07, $2016 \quad$ Published: January 23, 2017
}

Copyright: Zhong et al. This is an open-access article distributed under the terms of the Creative Commons Attribution License (CC-BY), which permits unrestricted use, distribution, and reproduction in any medium, provided the original author and source are credited.

\section{ABSTRACT}

Purpose: To evaluate the efficacy of IMRT combined with concurrent chemotherapy followed by adjuvant chemotherapy compared with IMRT combined with concurrent chemotherapy alone in patients with nasopharyngeal carcinoma.

Methods: From January 2007 to December 2014, we collected 797 staged II-IVb [UICC = Union for International Cancer Control criteria (7th edition)] NPC patients for analysis. After 1:1 matching, we selected 261 cases as the CCRT group, another 261 patients as the CCRT+AC group. Using Kaplan-Meier to calculate the overall survival (OS), locoregional failure-free survival(LFFS), distant metastasis failure-free survival(DMFS). The log-rank test and Cox-proportional hazards model to evaluate the prognostic factors.

Results: After matching, there were 261 patients in each group. In CCRT+AC group, The 1-,2- and 3- year os rates were a little higher than in CCRT group(99.6\% vs $97.9 \%, 97.4 \%$ vs $96.2 \%, 93.8 \%$ vs $86.9 \%, P=0.150)$. There were no significant difference in 1-,2-,3- year OS, LFFS, DMFS between the two groups. In subgroup analysis, a little higher OS rate in CCRT+AC group for staged III, IV and T4(III:100\% vs $100 \%, 97.6 \%$ vs $\mathbf{9 5 . 8 \% ,} 94.0 \%$ vs $84.0 \%$; IV: $\mathbf{9 9 . 1 \%}$ vs $95.4 \%$, $96.3 \%$ vs $95.4 \%$, $90.5 \%$ vs $79.4 \%, P=0.047 ; \mathrm{T} 4: 99.1 \%$ vs $95.2 \%, 97.1 \%$ vs $95.2 \%, 90.9 \%$ vs $78.2 \%$, $P=0.055)$. No significant difference were observed in OS, LFFS,DMFS between the groups.

Conclusion: IMRT combined with concurrent chemotherapy followed by adjuvant chemotherapy might improved 1-,2-,3- year of OS. Whether or not add adjuvant chemotherapy it had similar LFFS rate and DMFS rate in patients with nasopharyngeal carcinoma. Locally advanced NPC patients (III, IV and T4)might benefit from the adjuvant chemotherapy.

\section{INTRODUCTION}

Nasopharyngeal carcinoma (NPC)is popular in China, especially in Guangdong, Guangxi, Hunan, Hainan et al. Radiation therapy is the major treatment for NPC. At present, the standard treatment for NPC is concurrent chemo-radiotherapy. Since 0099 trail [1] reported that conventional chemotherapy combined with concurrent chemotherapy plus adjuvant chemotherapy can improved the overall survival in NPC patients in 1998. This study made sure the efficacy in treated with NPC. Joseph Wee [2] also verified the results as similar as 0099 trail. At the same year, they found that chemotherapy can improve the control rate of distant metastasis . Based on those research, the treatment of conventional radiation therapy combined with concurrent chemotherapy followed by 
Table 1: Baseline patient characteristics in the pre-matching and post-matching cohort

\begin{tabular}{|c|c|c|c|c|c|c|}
\hline \multirow[b]{2}{*}{ group } & \multicolumn{3}{|c|}{ Pre- matching } & \multicolumn{3}{|c|}{ post- matching } \\
\hline & $\mathrm{CCRT}^{\mathrm{b}}$ & $\mathrm{CCRT}+\mathrm{AC} \mathrm{C}^{\mathrm{C}}$ & $P$ value & CCRT & $C R T+A C$ & $P$ value \\
\hline patients & 261 & 536 & & 261 & 261 & \\
\hline age (year) & $47(22-73)$ & $44(16-75)$ & 0.060 & $47(22-73)$ & $44(16-75)$ & 0.191 \\
\hline sex & & & 0.175 & & & 0.250 \\
\hline male & $185(70.9)$ & $404(75.4)$ & & $185(70.9)$ & $189(72.4)$ & \\
\hline female & $76(29.1)$ & $132(24.6)$ & & $76(29.1)$ & $72(27.6)$ & \\
\hline overall stage $^{a}$ & & & 0.251 & & & 1.000 \\
\hline II & $37(14.2)$ & $73(13.6)$ & & $37(14.2)$ & $37(14.2)$ & \\
\hline III & $103(39.5)$ & $244(45.5)$ & & $103(39.5)$ & $103(39.5)$ & \\
\hline IV & $121(46.4)$ & $219(40.9)$ & & $121(46.4)$ & $121(46.4)$ & \\
\hline T classification ${ }^{a}$ & & & 0.274 & & & 1.000 \\
\hline $\mathrm{T} 1$ & $14(5.4)$ & $36(6.7)$ & & $14(5.4)$ & $14(5.4)$ & \\
\hline $\mathrm{T} 2$ & $57(21.8)$ & $115(21.5)$ & & $57(21.8)$ & $57(21.8)$ & \\
\hline T3 & $76(29.1)$ & $185(34.5)$ & & $76(29.1)$ & $76(29.1)$ & \\
\hline $\mathrm{T} 4$ & $114(43.7)$ & $200(37.3)$ & & $114(43.7)$ & $114(43.7)$ & \\
\hline $\mathrm{N}$ classification ${ }^{\mathrm{a}}$ & & & 0.939 & & & 1.000 \\
\hline NO & $12(4.6)$ & $25(4.7)$ & & $12(4.6)$ & $12(4.6)$ & \\
\hline N1 & $95(36.4)$ & $198(36.9)$ & & $95(36.4)$ & $95(36.4)$ & \\
\hline N2 & $141(54.0)$ & ) $281(52.4)$ & & $141(54.0)$ & 141 & \\
\hline N3 & $13(5.0)$ & $32(6.0)$ & & $13(5.0)$ & $13(5.0)$ & \\
\hline $\mathrm{KPS}^{\mathrm{d}}$ & & & 0.004 & & & 1.000 \\
\hline $90-100$ & $191(73.2)$ & ) $337(62.9)$ & & $191(73.2)$ & $191(73.2)$ & \\
\hline $70-80$ & $70(26.8)$ & 199 (37.1) & & $70(26.8)$ & $70(26.8)$ & \\
\hline
\end{tabular}

a By Union for International Cancer Control criteria (7th edition).

$\mathrm{b}$ CCRT=concurrent chemoradiotherapy

c $\mathrm{AC}=$ adjuvant chemotherapy

$\mathrm{d}$ KPS $=$ Karnofsky performance status

The method of matching, first is selected a total of 261 CCRT patients, then matching 1:1 on age, sex, KPS, overall stage, T classification, $\mathrm{N}$ classification with the CCRT group, selected the satisfactory patients from 536 patients in CCRT + AC.

adjuvant chemotherapy became a standard method to treat locoregionally advanced nasopharyngeal carcinoma at North America. But recently, a number of Meta analysis $[3,4]$ showed that, concurrent chemo-radiotherapy plus adjuvant chemotherapy could not improved the survival rate and most of researches were based on conventional radiotherapy. Now coming into the IMRT era, the problem whether or not adjuvant chemotherapy should be given after finished the concurrent chemo-radiotherapy stills controversial. So we collected 522 staged II-IVb NPC patients who received IMRT combined with concurrent chemotherapy followed by adjuvant chemotherapy or only received IMRT combined with concurrent chemotherapy, to investigate the value of adjuvant chemotherapy in NPC treatment.

\section{MATERIALS AND METHODS}

\section{Patients}

We retrospectively collected a total of 797 stagedII$\mathrm{IVb}[\mathrm{UICC}=($ Union for International Cancer Control criteria (7th edition)] NPC patients at the department of radiation oncology of tumor hospital affiliated with Guangxi Medical University, Nanning PR China, from January 2007 to December 2014. Included 261 cases treated with CCRT and 536 patients treated with CCRT+AC. The inclusion criteria:(1) All the patients were initial treatment who never received any chemotherapy or 
Table 2: chemotherapy administrated to 522 NPC patients in the pro-merging groups

\begin{tabular}{|c|c|c|c|c|}
\hline \multirow{3}{*}{ Regimen } & \multirow{3}{*}{ Weeks } & \multicolumn{2}{|c|}{ Group } & \multirow{3}{*}{$P$ value } \\
\hline & & $\mathrm{CCRT}^{\mathrm{C}}$ & $C C R T+A C^{d}$ & \\
\hline & & $(n=261)$ & $(n=261)$ & \\
\hline \multirow{4}{*}{$\begin{array}{l}\text { Concurrent chemotherapy } \\
\qquad \mathrm{DDP}^{\mathrm{a}}\end{array}$} & & & & 0.001 \\
\hline & 1 & 17 & 11 & \\
\hline & 2 & 77 & 119 & \\
\hline & 3 & 167 & 131 & \\
\hline \multicolumn{5}{|l|}{ Adjuvant chemotherapy } \\
\hline \multirow[t]{3}{*}{$\mathrm{PF}^{\mathrm{b}}$} & 1 & - & 70 & \\
\hline & 2 & - & 152 & \\
\hline & 3 & - & 39 & \\
\hline
\end{tabular}

a $\mathrm{DDP}=$ Cisplatin alone

b $\mathrm{PF}=$ Cisplatin plus 5-fluorouracil

c CCRT=concurrent chemoradiotherapy

$\mathrm{d} \mathrm{AC}=$ adjuvant cbhemotherapy

radiotherapy;(2)patients aged ranged from 16 to 75 with no distant metastasis ;(3)KPS $\geq 70$;(4) pathologic type was non-keratinizing and undifferentiated carcinoma. Those who had radiotherapy, chemotherapy or surgery to the head and neck,or those patients who had double cancer, serious heart and lung diseases, distant metastasis before treatment were exclusion. Before treated, all the patients had a medical history, physical examination, assessment of performance status, complete blood cell count, full bio-chemical profile, EB-virus DNA copies, electrocardiogram, chest computerized tomography (CT), abdominal ultrasound, magnetic resonance imaging (MRI) for nasopharyngeal and neck, nasopharyngeal fiber optic endoscopy and bone scan. In CCRT+AC group ,all the participants were received cisplatin plus 5-fluorouracil (PF) as the pattern of adjuvant chemotherapy .Because the KPS of baseline between the two groups were statistically significant difference. So we selected totally 261 patients who received CCRT, we matched 1:1 with the CCRT group on age, sex, overall stage, T classification, $\mathrm{N}$ classification, KPS, as the CCRT $+\mathrm{AC}$ group. After matched ,the baseline data of the two groups balance. (Table 1)

\section{Radiotherapy}

All enrolled patients were received intensitymodulated radiotherapy(IMRT) technique what was administered by a $6 \mathrm{MV}-\mathrm{X}$ ray liner accelerator. Patients were immobilized with a head- -neck-shoulder thermoplastic mask in the supine position. Then a CT simulation was carried out to scan the head and neck from the bottom-up. The scan range from the vertex to $2 \mathrm{~cm}$ below the clavicle. The slice extending from $2 \mathrm{~cm}$ over the anterior clinoid process to the level of cartilagines thyreoidea. The slice thickness was $2.5 \mathrm{~mm}$, apart from
$2.5 \mathrm{~mm}$, the remaining were $5 \mathrm{~mm}$. We use Precise Plan 2.11(Elekta,Crawley,UK)he Philips pinnacle v8.0(Philips Medical Systems,Milpitas,CA) to delineat the target volumes. The target volumes were delineated according to the International Commission on Radiation Units and Measurements Reports (ICRU)50 and 62.Gross tumor volume in the nasopharynx (GTVnx) stands for what we saw the gross tumor from the clinical examination and imaging examination. Gross

tumour volume of involved lymph nodes(GTVnd) was the positive lymph nodes. Clinical tumor volumes one(CTV1) was the high risk clinical tumor volumes , including GTV plus 5-10mm margin and metastasis lymph nodes. Clinical tumor volumes two (CTV2)was the low risk clinical tumor volumes, including the lymphatic regions and CTV1 plus 5-10mm margins. Planning target volumes(PTV) stand for a $3 \mathrm{~mm}$ margins were added to the GTV and the CTV. The total radiation prescribed dose was 68-74Gy/30-33 fractions to the PGTVnx,60-71Gy/30-33 fractions to the PGTVnd,60-66Gy/28-30 fractions to the PCTV1, 50-60Gy/28-30 fractions to the PCTV2 at 5 fractions per week during a period of 6-7 weeks.

\section{Chemotherapy}

Concurrent chemotherapy :1 to 3 cycles of Cisplatin alone $100 \mathrm{mg} / \mathrm{m} 2$ every 3 weeks;

Adjuvant chemotherapy: 1 to 3 cycles of Cisplatin $80 \mathrm{mg} / \mathrm{m} 2$ on day 1 to day 3 and 5 -fluorouracil( $5-\mathrm{Fu}) 750$ $\mathrm{mg} / \mathrm{m} 2 / \mathrm{d}$ on day 1 to day 5 (or continuous intravenous infusion for 120 hours) every 3 weeks(Table 2);

But the $\mathrm{p}$ value of chemotherapy regimen between two groups had significantly difference ,so we merged patients who receiving 2 cycles with 3 cycles in order to reduce the errors.(Table 3 ) 
Table 3: chemotherapy administrated to 522 NPC patients in the post-merging groups

\begin{tabular}{|c|c|c|c|c|}
\hline \multirow{3}{*}{ Regimen } & \multirow{3}{*}{ Weeks } & \multicolumn{2}{|c|}{ Group } & \multirow{3}{*}{ Pvalue } \\
\hline & & $\mathrm{CCRT}^{\mathrm{C}}$ & $C C R T+A C^{d}$ & \\
\hline & & $(n=261)$ & $(n=261)$ & \\
\hline \multirow{3}{*}{$\begin{array}{l}\text { Concurrent chemotherapy } \\
\qquad \mathrm{DDP}^{\mathrm{a}}\end{array}$} & & & & 0.244 \\
\hline & 1 & 17 & 11 & \\
\hline & $2-3$ & 244 & 250 & \\
\hline \multicolumn{5}{|l|}{ Adjuvant chemotherapy } \\
\hline \multirow[t]{3}{*}{$\mathrm{PF}^{\mathrm{b}}$} & 1 & - & 70 & \\
\hline & 2 & - & 152 & \\
\hline & 3 & - & 39 & \\
\hline
\end{tabular}

a DDP $=$ Cisplatin alone

b $\mathrm{PF}=$ Cisplatin plus 5-fluorouracil

c CCRT=concurrent chemoradiotherapy

$\mathrm{d} \mathrm{AC}=$ adjuvant cbhemotherapy

Table 4: multivariate Cox regression analysis of the prognostic factors

\begin{tabular}{|c|c|c|c|c|c|c|c|c|c|c|c|c|}
\hline & \multicolumn{4}{|c|}{ OS } & \multicolumn{3}{|c|}{ LFFS } & \multicolumn{4}{|c|}{ DMFS } & \multirow{3}{*}{$P$ value } \\
\hline & \multirow[t]{2}{*}{ HR } & \multicolumn{2}{|c|}{$95 \% \mathrm{Cl}$} & \multirow[t]{2}{*}{$P$ value } & \multirow[t]{2}{*}{ HR } & \multicolumn{2}{|c|}{$95 \% \mathrm{Cl}$} & \multirow[t]{2}{*}{$P$ value } & \multirow[t]{2}{*}{ HR } & \multicolumn{2}{|c|}{$95 \% \mathrm{Cl}$} & \\
\hline & & Lower & upper & & & Lower & upper & & & Lower & upper & \\
\hline Age & 1.013 & 0.981 & 1.040 & 0.423 & 0.977 & 0.939 & 1.018 & 0.271 & 0.994 & 0.967 & 1.023 & 0.702 \\
\hline KPS & 0.777 & 0.356 & 1.697 & 0.526 & 0.753 & 0.268 & 2.117 & 0.590 & 0.770 & 0.380 & 1.559 & 0.468 \\
\hline Overall Stage & 2.007 & 0.550 & 7.325 & 0.291 & 1.896 & 0.366 & 9.820 & 0.446 & 1.684 & 0.599 & 4.732 & 0.323 \\
\hline Course Of con-chemo ${ }^{a}$ & 0.588 & 0.329 & 1.050 & 0.073 & 0.882 & 0.421 & 1.849 & 0.740 & 0.527 & 0.327 & 0.848 & 0.008 \\
\hline Course Of adj-chemo ${ }^{b}$ & 0.814 & 0.567 & 1.166 & 0.261 & 1.096 & 0.697 & 1.722 & 0.692 & 0.968 & 0.719 & 1.304 & 0.830 \\
\hline
\end{tabular}

$\mathrm{a}=$ Course Of con-chemotherapy

$b=$ Course of adjuvant chemotherapy

\section{Follow-up}

After all treatment completion, all patients were subsequently followed up every 3 months for the first two years . Every 6 months through the following 3 to 5 years, and then per annum. The follow-up content including body check, complete blood cell count, liver and kidney founctions, EB virus DNA copies, chest computerized tomography (CT), abdominal ultrasound, magnetic resonance imaging (MRI) for nasopharyngeal and neck, nasopharyngeal fiber optic endoscopy and bone scan. The primary endpoint was OS,LFFS,DMFS. The whole group median follow-up period was 25.65 months( range, 2.1398.70 months).

\section{Statistical analysis}

All data were analyzed by the statistical package for Social Sciences, version 19.0 (SPSS,Chicago, IL.USA). We measured the survival time from the first day the patient diagnosed NPC to the day of the event. The $\chi^{2}$ test was used to compare the based-line data(e.g: sex, KPS, clinical stage, $\mathrm{T}$ classification ,N classification). For those small sample data we used the fisher's exact test. Using independent-samples $\mathrm{T}$ test to compare the age. The Kaplan-Meier method was used to calculate the OS,LFFS and DMFS rates. The log-rank test was used to compare the statistical differences in endpoints in both groups. Using Cox proportional hazards model to calculate the Multivariate analysis. All statistical tests were two-sided, and $\mathrm{P}<0.05$ was considered statistically significant .

\section{RESULTS}

\section{Patient outcomes}

OS

Before matching, there were 58 patients dead in the whole group, including 14 of the CCRT group and 44 of the CCRT+AC group. After matching, there were 32 patients dead in the whole group, including 14 patients in the CCRT group and 18 in the CCRT $+\mathrm{AC}$ group. The 
Table 5: The results from Stratified analysis in CCRT group and CCRT+AC group

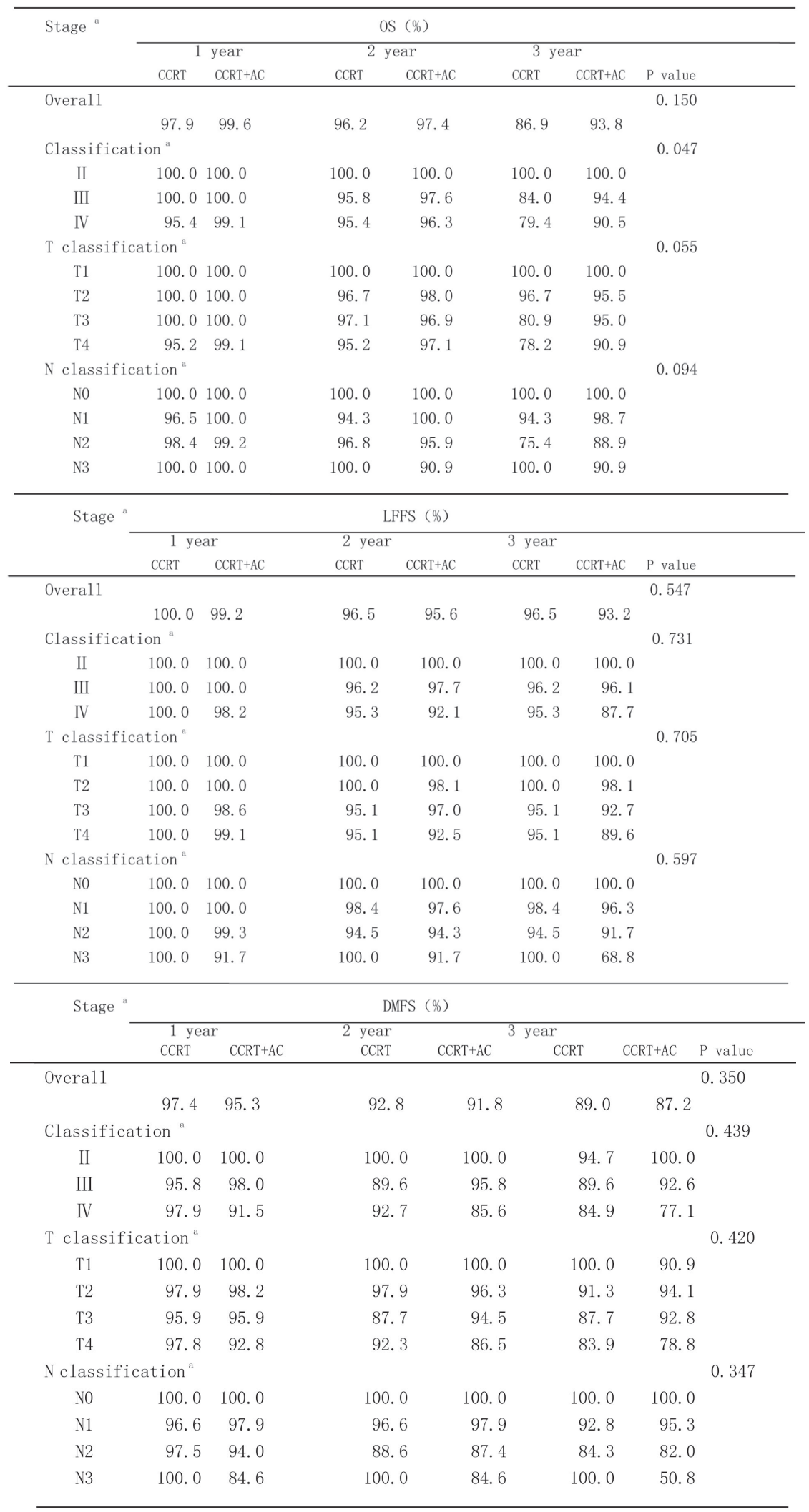

$\mathrm{a}=$ By Union for International Cancer Control criteria (7th edition)

Abbreviations: OS, overall survival; LFFS, locoregional failure-free survival; DMFS, distant metastasis failure-free survival; CCRT, concurrent chemoradiotherapy; AC, adjuvant chemotherapy 
Table 6: The distribution of 42 NPC patients with distant metastasis

\begin{tabular}{lcc}
\hline distant metastasis & $\begin{array}{c}\text { CCRT } \\
(\mathrm{n}=14)\end{array}$ & $\begin{array}{c}\text { CCRT }+\mathrm{AC} \\
(\mathrm{n}=28)\end{array}$ \\
\hline lung & 5 & 7 \\
bone & 2 & 7 \\
brain, bone and lung & 2 & 4 \\
lung and bone & 1 & 4 \\
liver & 2 & 3 \\
lung and liver & 1 & 0 \\
bone and liver & 0 & 1 \\
bone and brain & 1 & 0 \\
lung, bone and liver & 0 & 2 \\
\hline
\end{tabular}

1-,2-,3-year overall survival rates were 97.9\%, 96.2\%, $86.9 \%(95 \%$ CI 82-94),respectively in CCRT group versus $99.6 \%, 97.4 \%, 93.8 \%(95 \%$ CI $77-82)$ in CCRT $+\mathrm{AC}$ group. There were no significant difference in OS between the two groups (HR 0.595, CI 0.291-1.215, $\chi^{2}=2.072, P$ $=0.150)$ (Figure 1 and 4). After stratification by disease stage[UICC(7th edition)], the 1,2 and 3 years OS rates were all $100.0 \%$ in both groups for stage II. For stage III, the the 1,2 and 3 years os rates were $100.0 \%, 95.8 \%, 84.0 \%$ in CCRT group, while in CCRT $+\mathrm{AC}$ group ,the 1-,2- and 3year os rates were $100.0 \%, 97.6 \%, 94.4 \%$. For stage IV, the
1-,2- and 3- year os were 95.4\%,95.4\%,79.4\%,respectively in CCRT group versus 99.1\%, 96.3\%,90.5\% in CCRT+AC group. There were statistically significant difference in the whole group(HR 0.482, CI 0.232-1.004, $\chi^{2}=3.931, P=$ 0.047).However, after we pairwise for each stratum, there were no statistically significant difference for stage III and IV in 1-,2- and 3- year os rates $\left(\chi^{2}=1.811, P=0.178 ; \chi^{2}\right.$ $=2.168, P=0.141)$ between the two groups. For T1, the 1-,2- and 3- year os rates were all $100.0 \%$ between the two groups. For T2, the 1-,2- and 3- year os rates were $100.0 \%, 96.7 \%, 96.7 \%$ in CCRT group versus $100.0 \%$,

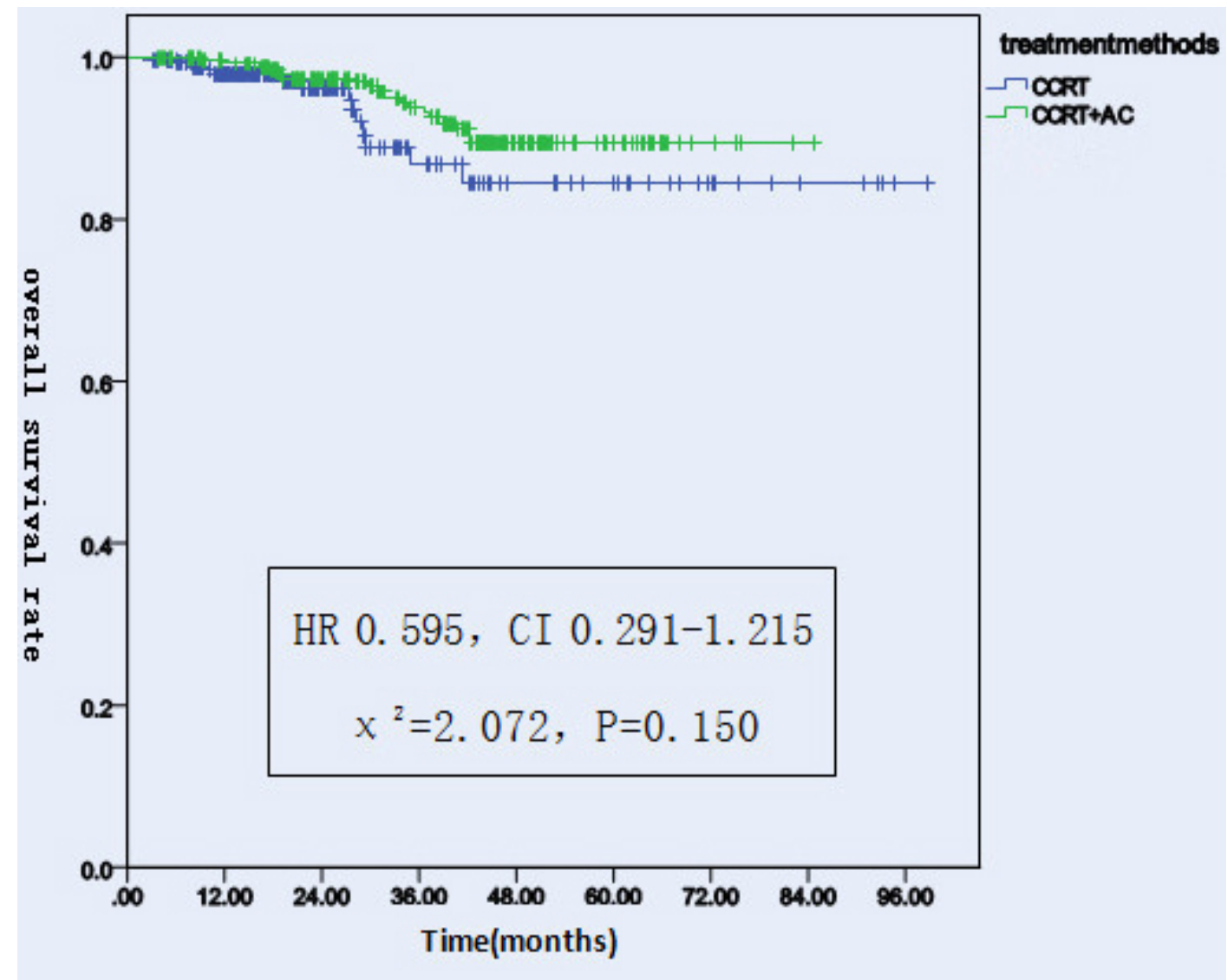

Figure 1: Overall survival rates of 522 NPC patients treated with different methods. 
98.0\%, 95.5\% in CCRT+AC group. For T3, the 1,2, and 3 years os rates were $100.0 \%, 97.1 \%, 80.9 \%$ in CCRT group, while in CCRT+AC group the 1,2,and 3 years os rates were $100.0 \%, 96.9 \%, 95.0 \%$.For T 4 , the 1,2 , and 3 years os rates were $95.2 \%, 95.2 \%, 78.2 \%$ in CCRT group versus $99.1 \%, 97.1 \%, 90.9 \%$ in CCRT + AC group. There were no significant difference in os of the whole group (HR 0.494, CI 0.238-1.027, $\chi^{2}=3.682, P=0.055$ ). For N0, there were $100.0 \%$ of the $1,2,3$ years os rates in the two groups. For N1,the 1-, 2- , 3- year os rates were 96.5\%, 94.3\%, 94.3\% in CCRT group, while in CCRT+AC group there were $100.0 \%, 100.0 \%, 98.7 \%$,respectively. For N2, the 1-, 2- , 3- year os rates were $98.4 \%, 96.8 \%, 75.4 \%$ in CCRT group versus $99.2 \%, 95.9 \%, 88.9 \%$ in the CCRT + AC group. For N3, the 1-, 2- , 3- year os rates were $100.0 \%$ in CCRT group versus $100.0 \%, 90.9 \%, 90.9 \%$ in CCRT + AC group. There were no significant difference in the whole group (HR 0.543, CI 0.263-1.121, $\chi^{2}=2.803, P$ $=0.094$, Table 5).

\section{LFFS}

There were 20 patients had localregional failure, including 6 patients in CCRT group and 14 patients in CCRT + AC group. Among them, 5 patients relapsed at the neck, the others all relapsed at nasopharynx . The 1, 2, 3 years LFFS rates were $100.0 \%, 96.5 \%, 96.5 \%(95 \%$ CI 91-98) in the CCRT group versus $99.2 \%, 95.6 \%, 93.2 \%$ (95\% CI 78-83)in the CCRT+AC group. There were no significant difference between the two groups(HR 1.345, CI $0.512-3.535, \chi^{2}=0.363, P=0.547$ ) (Figure 2 and 5). After stratification by disease stage[UICC(7th edition)]. In CCRT group ,the 1,2 and 3 years LFFS rates were all $100.0 \%$ for stage II, $100.0 \%, 96.2 \%, 96.2 \%$, for stage III, $100.0 \%, 95.3 \%, 95.3 \%$ for stage IV,respectively .On the contrary, in CCRT+AC group, the 1-,2-,3-year LFFS rates were all $100.0 \%$ for stage II, $100.0 \%, 97.7 \%, 96.1 \%$ for stage III, $98.2 \%, 92.1 \%, 87.7 \%$ for stage IV,respectively. There were no statistically significant difference in the whole group (HR 1.187, CI 0.446-3.160, $\chi^{2}=0.118, P$ $=0.731$ ). For T1, both groups were $100.0 \%$ of the 1 -, $2-$, and 3-year LFFS rates. For T2, the 1-, 2-, and 3-year LFFS rates were all $100.0 \%$ in CCRT group versus $100.0 \%, 98.1 \%, 98.1 \%$ in CCRT + AC group. For T3, the 1-, 2-, and 3-year LFFS rates were 100.0\%, 95.1\%, $95.1 \%$ in CCRT group versus $98.6 \%, 97.0 \%, 92.7 \%$ in CCRT + AC group. For T4, the 1,2 and 3 years LFFS rates were $100.0 \%, 95.1 \%, 95.1 \%$ in CCRT group ,respectively. While in CCRT $+\mathrm{AC}$ group there were $99.1 \%, 92.5 \%$,

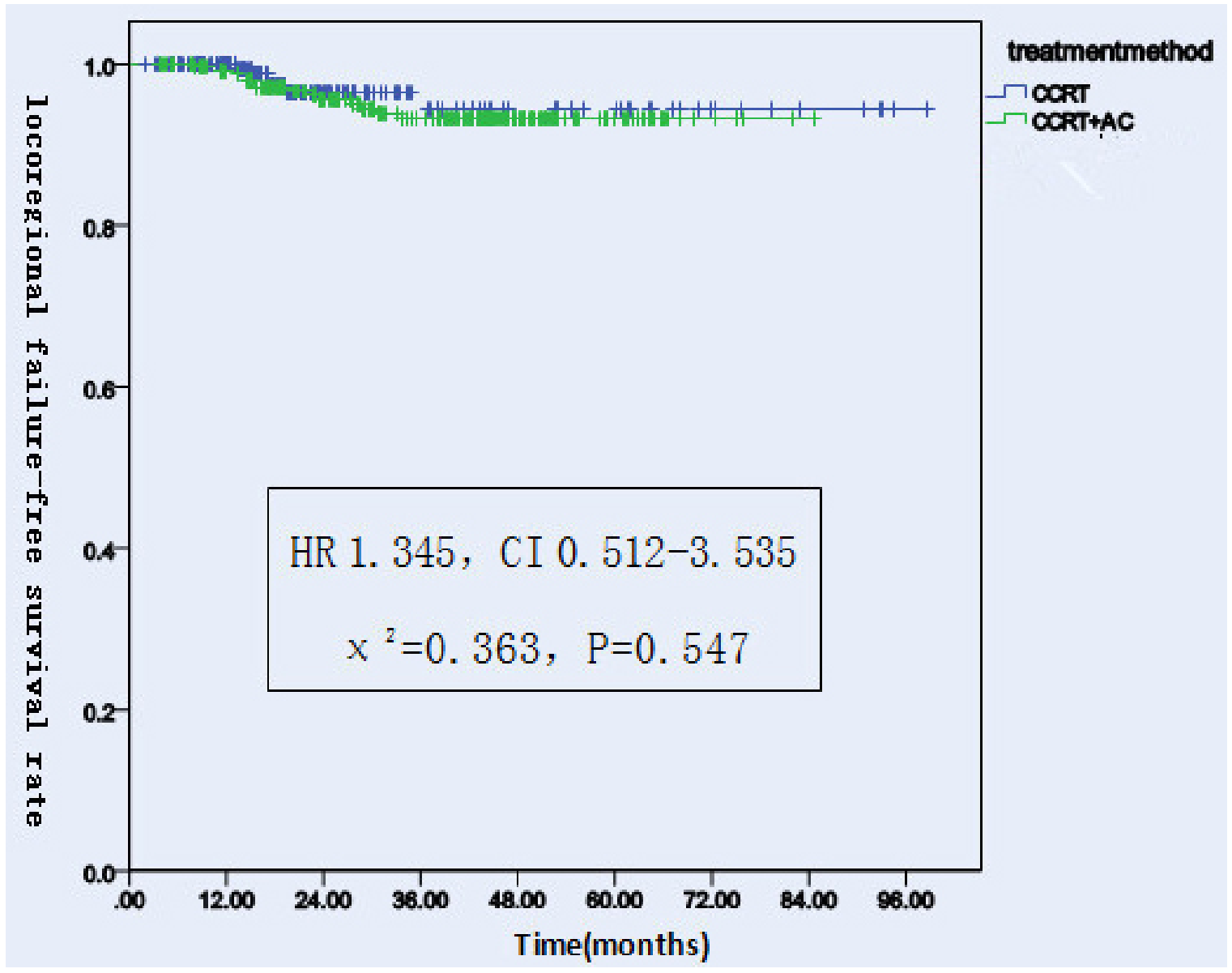

Figure 2: Locoregional failure-free survival rates of 522 NPC patients treated with different methods. 
$89.6 \%$, respectively. There were no statistically significant difference in the whole group (HR 1.207, CI 0.455$\left.3.204, \chi^{2}=0.143, P=0.705\right)$. In CCRT group , there were $100.0 \%, 100.0 \%, 100.0 \%$ of the 1-,2-,3-year LFFS rates for N0. $100.0 \%, 98.4 \%, 98.4 \%$ for N1. $100.0 \%, 94.5 \%, 94.5 \%$ for $\mathrm{N} 2.100 .0 \%, 100.0 \%, 100.0 \%$ for N3. In CCRT+AC group, the 1-,2-,3-year LFFS rates were all $100.0 \%$ for N0. $100.0 \%, 97.6 \%, 96.3 \%$ for N1. $99.3 \%, 94.3 \%, 91.7 \%$ for $\mathrm{N} 2.91 .7 \%, 91.7 \%, 68.8 \%$ for $\mathrm{N} 3$. There were no significant difference in the whole group(HR 1.301, CI $0.489-3.462, \chi^{2}=0.279, P=0.597$, Table 5).

\section{DMFS}

There were 42 patients had distant metastasis, including 14 patients in CCRT group and 28 patients in $\mathrm{CCRT}+\mathrm{AC}$ group. Among them, there were 12 cases had distant metastasis in lung, 9 cases in bone, 6 cases in brain, bone and lung, 5 cases both in lung and bone, 5 cases in liver, 1 case both in lung and liver, 1 case in bone and brain, 2 cases in lung, bone and liver (Table 6) . The 1-,2-,3-year MDFS rates were 97.4\%, 92.8\%, 89.0\% (95\% CI 85-95)in CCRT group. While in CCRT + AC group the 1-,2-,3-year DMFS rates were 95.3\%, 91.8\%, 87.2\%(95\% CI 73-79). There were no significantly difference in the two groups (HR 1.363, CI 0.711-2.612, $\chi^{2}=0.875, P$ $=0.350$, Figure 3 and 6 ). After stratification by disease
stage[UICC(7th edition)], the 1,2, and 3 years DMFS rates were $100.0 \%, 100.0 \%, 94.7 \%$ in CCRT group versus $100.0 \%, 100.0 \%, 100.0 \%$ in CCRT + AC group For stage II. For stage III,the 1,2,and 3 years DMFS rates were $95.8 \%, 89.6 \%, 89.6 \%$ in CCRT group versus $98.0 \%$, $95.8 \%, 92.6 \%$ in CCRT + AC group. For stage IV,the 1,2 , and 3 years DMFS rates were $97.9 \%, 92.7 \%, 84.9 \%$ in CCRT group versus $91.5 \%, 85.6 \%, 77.1 \%$ in CCRT + AC group. There were no statistically significant difference in the whole group(HR 1.295, CI 0.672-2.495, $\chi^{2}=0.600$, $P=0.439$ ).In CCRT group, the 1,2 , and 3 years DMFS rates were all $100.0 \%$ for $\mathrm{T} 1.97 .9 \%, 97.9 \%, 91.3 \%$ for T2. $95.9 \%, 87.7 \%, 87.7 \%$ for T3. $97.8 \%, 92.3 \%, 83.9 \%$ for T4,respectively. While in the CCRT+AC group, the comparable rates were $100.0 \%, 100.0 \%, 90.9 \%$ for $\mathrm{T} 1$. $98.2 \%, 96.3 \%, 94.1 \%$ for T2. $95.9 \%, 94.5 \%, 92.8 \%$ for T3. 92.8\%, 86.5\%, 78.8\% for T4,respectively. There were no statistically significant difference in the results (HR 1.309, CI 0.680-2.521, $\left.\chi^{2}=0.651, P=0.420\right)$. In CCRT group, the 1,2, and 3 years DMFS rates were all $100.0 \%$ for N0. $96.6 \%, 96.6 \%, 92.8 \%$ for N1. $97.5 \%, 88.6 \%, 84.3 \%$ for $\mathrm{N} 2.100 .0 \%, 100.0 \%, 100.0 \%$ for N3.But in CCRT $+\mathrm{AC}$ group, the comparable rates were all $100.0 \%$ for $\mathrm{N} 0$. $97.9 \%, 97.9 \%, 95.3 \%$ for N1. $94.0 \%, 87.4 \%, 82.0 \%$ for N2. $84.6 \%, 84.6 \%, 50.8 \%$ for N3,respectively. There were no significantly difference in that results (HR 1.366, CI

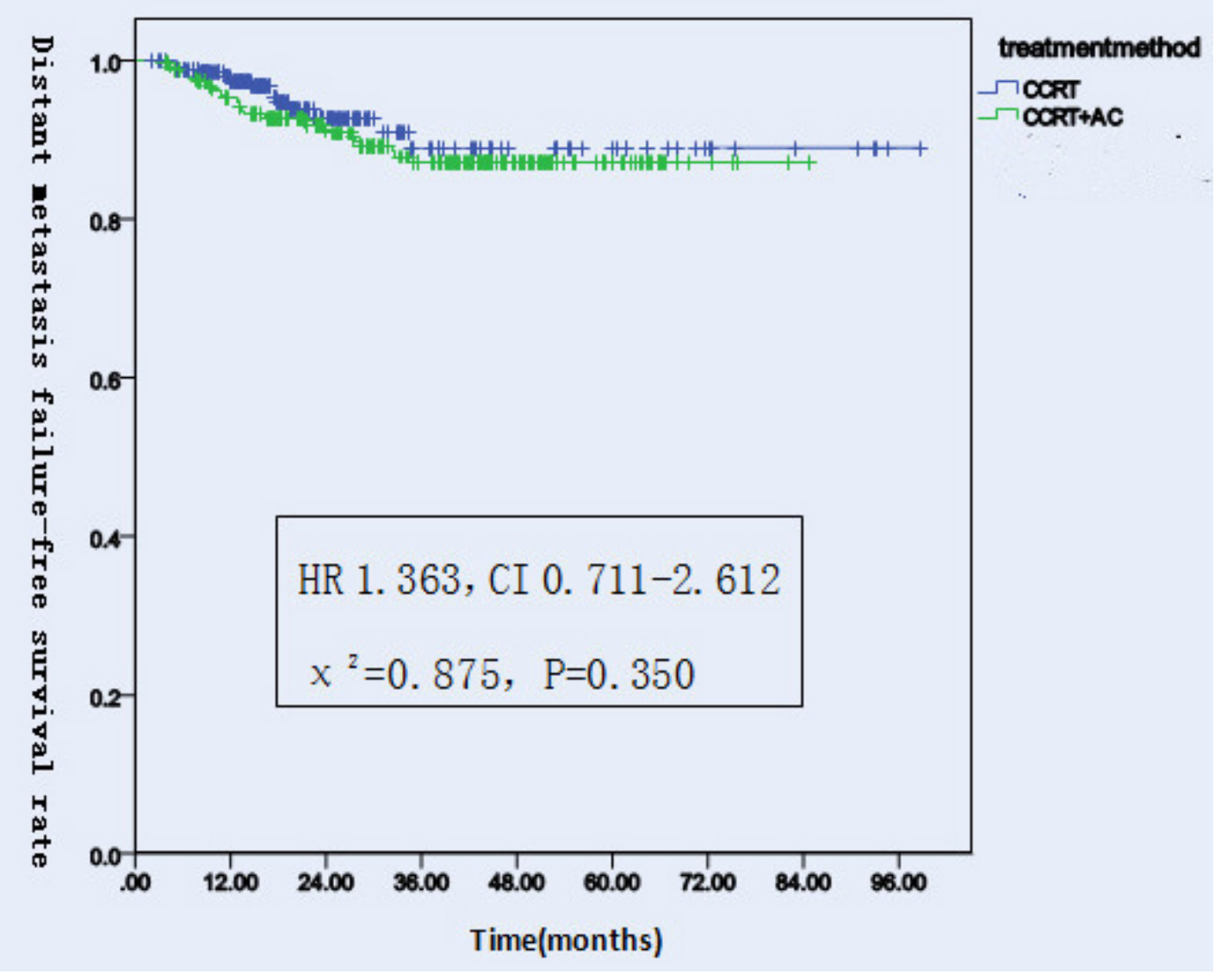

Figure 3: Distant metastasis failure-free survival rates of 522 NPC patients treated with different methods. 


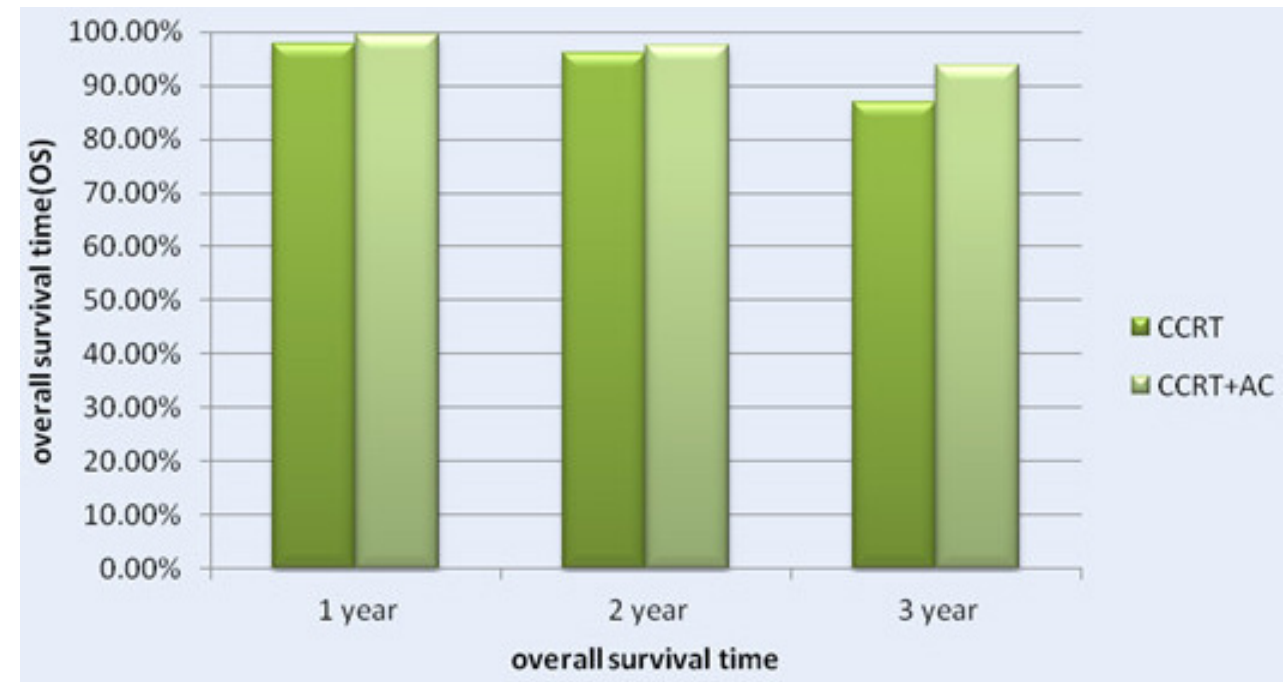

Figure 4: The 1-, 2- and 3- Year Overall Survival Rate (OS) for Patients with CCRT in Comparison with CCRT + AC for NPC patients.

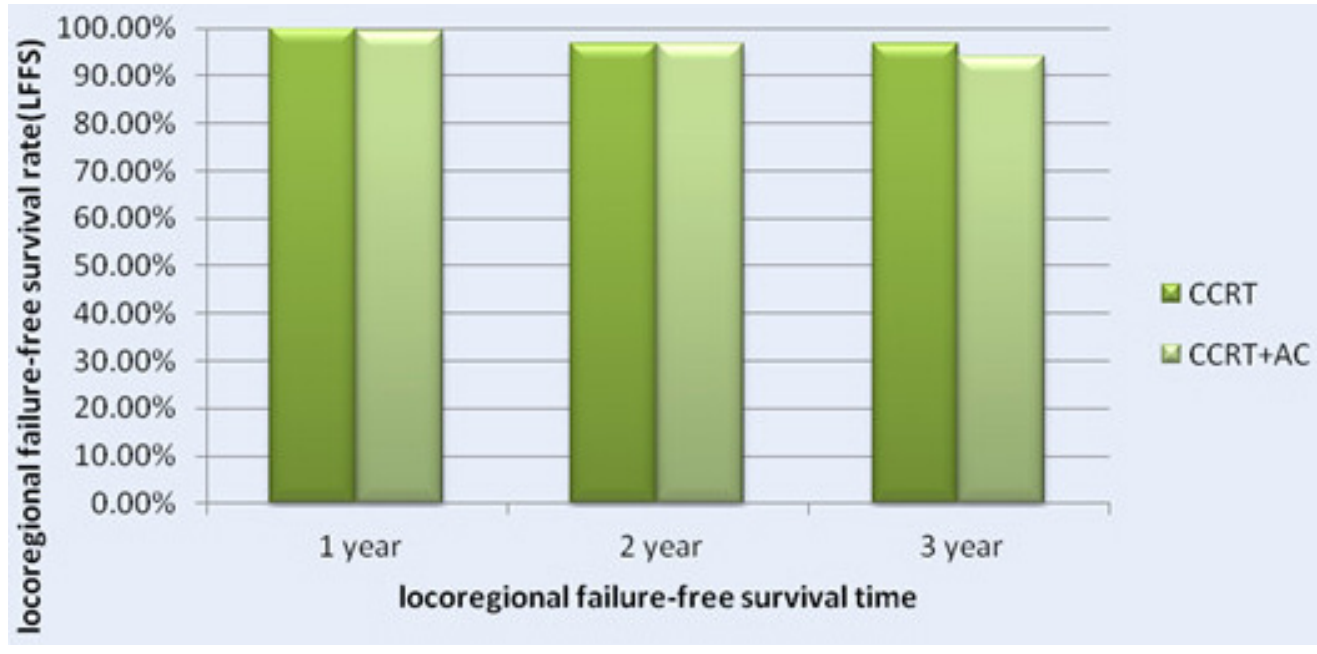

Figure 5: The 1-, 2- and 3- Year locoregional failure-free survival rate (LFFS) for Patients with CCRT in Comparison with CCRT + AC for NPC patients.

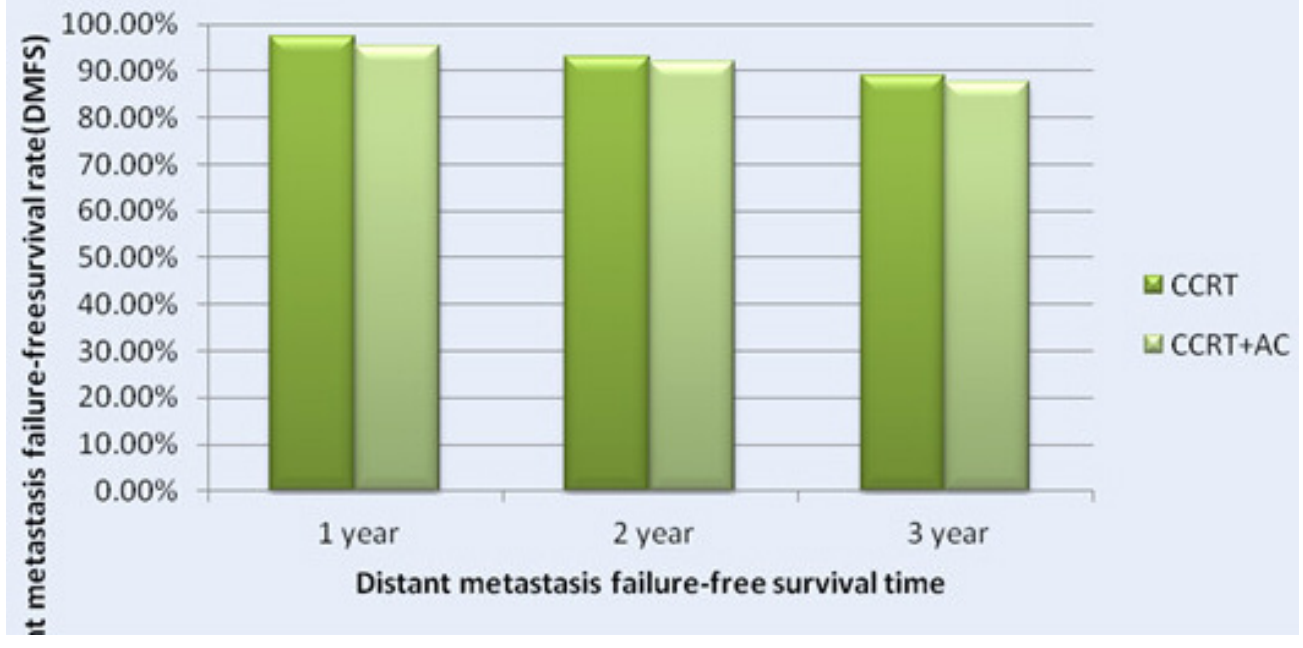

Figure 6: The 1-, 2- and 3- Year distant metastasis failure-free survival rate (DMFS) for Patients with CCRT in Comparison with CCRT + AC for NPC patients. 
$0.711-2.623, \chi^{2}=0.883, P=0.347$, Table 5).

\section{Multivariate analyses}

Multivariate Analyses by Cox proportional hazards model revealed that age, KPS scores, overall stage, treatment method and chemotherapy regimen were not the prognostic factors for OS. Only course of the concurrent chemotherapy was the prognostic factors for DMSF(Table 4).

\section{DISCUSSION}

In the age of two-dimensional radiotherapy, the five-year overall survival rate had a good efficiency in NPC [5], but it brought heavier sequelae and more chances to get radio-encephalopathy, patients had low quality of life. Since intensity modulated radiotherapy technique arose, because its targeted volume is closer to the tumor's shape, and it has lower injury to proximal critical normal tissues. So it gradually replace the twodimensional radiotherapy, becoming a principal treatment method for NPC patients. Zhang et al [6] made a study in the application of two-dimensional radiotherapy and IMRT in NPC patients, the results showed that using IMRT can obviously relieved acute or chronic radiation damage, and it had lower chance to have radio-sequelae than using two-dimensional radiotherapy, but IMRT could not improve distant metastasis-free rate, failure-free survival rate and overall survival rate. Later, Lai et al. [7] make a comparison between IMRT and two-dimensional radiotherapy in NPC. They found that IMRT significantly improved the treatment efficacy, patients achieved a good regional control rate, especially for those patients who had early T stage. In 2004, a multi-institutional survey of the effectiveness of chemotherapy plus radiotherapy for NPC by Mitsuhiko Kawashima [8] showed that, concurrent chemotherapy combine with radiotherapy could significantly improved the overall survival rate than only received radiotherapy in advanced NPC patients. A great deal of studies $[9,10,20]$ had approved concurrent chemoradiotherapy had advantages in improving overall survival rate and progression free survival rate, what indicated that locally advanced NPC patients received concurrent chemoradiotherapy would more beneficial. So in 2010, National Comprehensive Cancer Network (NCCN) recommended concurrent chemoradiotherapy was the standard treatment method for NPC. In 2014, Tingting $\mathrm{Xu}$ et al [11] make a comparison between concurrent chemoradiotherapy and neoadjuvant chemotherapy for locally advanced NPC patients. The results pointed out both regimens showed similar efficacy. Only concurrent chemotherapy could ameliorate distant metastasis free survival rate for those stage T3-4N0-1 NPC patients. In a trail by su [12], 865 NPC patients received IMRT alone or plus chemotherapy, they classified the candidates into four groups, From the study they made a conclusion that the early disease group had the lowest treatment failure rate. For those NPC patients with early stage, IMRT alone could produce satisfactory results. While for those patients who had locally advanced, chemoradiotherapy was better. Early in 1988, Rossi A et al. [13] had a comparison between radiotherapy plus adjuvant chemotherapy and radiotherapy alone. They found there were no significantly difference in two-year progression-free survival rate and the four-year overall survival rate $(55.8 \%$ vs. $57.7 \%$, $67.3 \%$ vs. 58.5\%). In 2010, Kyong Hwa Park et al. [14] reported that the overall response rate of CCRT was $95 \%$, after used $\mathrm{AC}$, the overall response rate was $100 \%$. So they make a conclusion that using cisplatin and 5-FU in combination with radiotherapy followed by three cycles of BEC chemotherapy was effective in locally advanced NPC patients. In 2011, a study by Anne W.M. Lee et al. [15] showed that, concurrent chemoradiotherapy followed by adjuvant chemotherapy can significantly reduced treatment failure and cancer-specific deaths. Recently, a meta analysis by Marie Yan showed that concurrent chemoradiotherapy followed by adjuvant chemotherapy can't enhance survival rates. An other meta-analysis at the same year by Blanchard P et al. [4] found that, concurrent chemoradiotherapy were better than chemotherapy alone in improving survival rates. In our study, no significantly difference in 1-,2-,3-year OS, LFFS and DMFS between CCRT alone and CCRT + AC in NPC patients $\left(\chi^{2}=2.072, P\right.$ $=0.150 ; \chi^{2}=0.363, P=0.547 ; \chi^{2}=0.875, P=0.350$ ), what are similar to the results by Dora L.W. et al. [16] in 2004. Nevertheless, after stratification by disease stage, we found a statistically significant difference in $\operatorname{OS}(P=0.047)$. But after we pairwise for each stratum, there were no statistical significance for stage III and IV in two-year os rates $\left(\chi^{2}=\right.$ $\left.1.811, P=0.178 ; \chi^{2}=2.168, P=0.141\right)$. We thought it was due to confounding factors. Although other results from the Stratified analysis didn't make a statistically significant difference, we saw a trend that the CCRT+AC group has higher OS than CCRT group for locally advanced NPC(III,IV and T4)patients from the results .Adding adjuvant chemotherapy for locally advanced NPC patients may benefit. Furthermore, addition of AC couldn't improved the 1-,2-,3-year LFFS and DMFS for N2-3. On the contrary, it may reduced the 1-,2-,3-year LFFS rate or DMFS rate .This results compared with a study reported by liang [19] at 2014 has some inconsistencies .He reported that concurrent chemoradiotherapy plus adjuvant chemotherapy didn't significantly improve the 2-year OS,LFFS,DMFS, but he found a borderline significant difference in os by $\mathrm{CCRT}+\mathrm{AC}$ treatment in patients with N2-3 disease $(P=0.052)$. Analyzed its cause, the main possibility is we enlarged the number of participants. Why concurrent chemoradiotherapy followed by adjuvant chemotherapy may reduced the efficacy for N2-3 patients? One possibility is that for those advanced 
nodal NPC patients(N2-3), concurrent chemoradiotherapy also have a better efficacy, after chemoradiotherapy, local organizations fibrosis so that with poor blood supply, chemotherapy drugs hardly get into the tumor site, so the plasma concentration beyond the reach of effective concentration. Another possibility explanation is that stage N2-3 patients have poor immunity so they can't tolerant the chemotherapy. In a phase 3 multicentre randomised controlled trial reported by chen et al. [17], concurrent chemoradiotherapy followed by cisplatin and fluorouracil chemotherapy did not improve failurefree survival rate in locoregionally advanced NPC patients. The same conclusion by Shiping Yang [18], concurrent chemoradiotherapy plus adjuvant cisplatin or nedaplatin and 5-fluorouracil chemotherapy didn't significantly improve 3 years OS, LRFS,FFS,DMFS. In 2011, a trail reported by Anne W.M. Lee [21], concurrent chemoradiotherapy was important to locoregional control and survival. Moreover, they though 2 concurrent cycles of cisplatin were enough. Adjuvant chemotherapy using fluorouracil-containing combination can improved distant control. At present, it is uncertain whether concurrent chemoradiotherapy followed by chemotherapy have survival benefit.

Although our trail is a big data retrospective analysis, it has several limitations. The reliability needs more prospective studies to verify the conclusion.

\section{CONCLUSIONS}

From this study, IMRT combined with concurrent chemotherapy followed by adjuvant chemotherapy in NPC might improve the 1-,2-, and 3- year OS, but no significant difference were observed between the two groups. There was no statistically significant difference in LFFS and DMFS. For those locally advanced NPC patients(III,IV and T4) might benefit from adjuvant chemotherapy .

\section{COMPLIANCE WITH ETHICAL STANDARDS}

The study was approved by the Ethics Committee of the Cancer Hospital of Guangxi Medical University.

\section{CONFLICTS OF INTEREST}

The authors declare no financial conflicts of interest regarding the design or results of this study.

\section{REFERENCES}

1. Al-Sarraf M, LeBlanc M, Giri PG, Fu KK, Cooper J, Vuong T, Forastiere AA, Adams G, Sakr WA, Schuller DE, Ensley JF. Chemoradiotherapy versus radiotherapy in patients with advanced nasopharyngeal cancer: phase III randomized
Intergroup study 0099. J Clin Oncol. 1998; 16:1310-17.

2. Wee J, Tan EH, Tai BC, Wong HB, Leong SS, Tan T, Chua ET, Yang E, Lee KM, Fong KW, Tan HS, Lee KS, Loong S, et al. Randomized trial of radiotherapy versus concurrent chemoradiotherapy followed by adjuvant chemotherapy in patients with American Joint Committee on Cancer/International Union against cancer stage III and IV nasopharyngeal cancer of the endemic variety. J Clin Oncol. 2005; 23:6730-38.

3. Yan M, Kumachev A, Siu LL, Chan KK. Chemoradiotherapy regimens for locoregionally advanced nasopharyngeal carcinoma: A Bayesian network metaanalysis. Eur J Cancer. 2015; 51:1570-79.

4. Blanchard P, Lee A, Marguet S, Leclercq J, Ng WT, Ma J, Chan AT, Huang PY, Benhamou E, Zhu G, Chua DT, Chen Y, Mai HQ, et al. Chemotherapy and radiotherapy in nasopharyngeal carcinoma: an update of the MAC-NPC meta-analysis. Lancet Oncol. 2015; 16:645-55.

5. Li G, Junlin Y, Xiaodong H, Suyan L, Jingwei L, Guozhen $X$. Summary of ten years of radical radiotherapy for nasopharyngeal carcinoma .chinese journal of radiation oncology. 2006;15:249-256.

6. Zhang Y, Lin ZA, Pan JJ, Zheng Z, Yang L, Lin SJ, Zheng F. Concurrent control study of different radiotherapy for primary nasopharyngeal carcinoma: intensity-modulated radiotherapy versus conventional radiotherapy.chinese cancer. 2009 ;28:1143-1148.

7. Lai SZ, Li WF, Chen L, Luo W, Chen YY, Liu LZ, Sun Y, Lin AH, Liu MZ, Ma J. How dose intensitymodulated radiotherapy versus conventional twodimension radiotherapy influence the treatment results in nasopharyngeal carcinoma patients? Int J Radiat Oncol Biol Phys. 2011; 80:661-68.

8. Kawashima M, Fuwa N, Myojin M, Nakamura K, Toita T, Saijo S, Hayashi N, Ohnishi H, Shikama N, Kano M, Yamamoto M. A multi-institutional survey of the effectiveness of chemotherapy combined with radiotherapy for patients with nasopharyngeal carcinoma. Jpn J Clin Oncol. 2004; 34:569-83.

9. Al-Sarraf M, LeBlanc M, Giri PG, Fu KK, Cooper J, Vuong T, Forastiere AA, Adams G, Sakr WA, Schuller DE, Ensley JF. Chemoradiotherapy versus radiotherapy in patients with advanced nasopharyngeal cancer: phase III randomized Intergroup study 0099. J Clin Oncol. 1998; 16:1310-17.

10. Lee AW, Tung SY, Chua DT, Ngan RK, Chappell R, Tung R, Siu L, Ng WT, Sze WK, Au GK, Law SC, O’Sullivan $\mathrm{B}$, Yau TK, et al. Randomized trial of radiotherapy plus concurrent-adjuvant chemotherapy vs radiotherapy alone for regionally advanced nasopharyngeal carcinoma. J Natl Cancer Inst. 2010; 102:1188-98.

11. Xu T, Zhu G, He X, Ying H, Hu C. A phase III randomized study comparing neoadjuvant chemotherapy with concurrent chemotherapy combined with radiotherapy for locoregionally advanced nasopharyngeal carcinoma: 
updated long-term survival outcomes. Oral Oncol. 2014; 50:71-76.

12. Su SF, Han F, Zhao C, Huang Y, Chen CY, Xiao WW, Li JX, Lu TX. Treatment outcomes for different subgroups of nasopharyngeal carcinoma patients treated with intensitymodulated radiation therapy. Chin J Cancer. 2011; 30:56573.

13. Rossi A, Molinari R, Boracchi P, Del Vecchio M, Marubini E, Nava M, Morandi L, Zucali R, Pilotti S, Grandi C, Ambrosini G, Cellini N, Chiavacci A, et al. Adjuvant chemotherapy with vincristine, cyclophosphamide, and doxorubicin after radiotherapy in local-regional nasopharyngeal cancer: results of a 4-year multicenter randomized study. J Clin Oncol. 1988; 6:1401-10.

14. Park KH, Kim JS, Park Y, Seo HY, Park YJ, Choi IK, Oh SC, Seo JH, Kim CY, Jung KY, Shin SW, Kim YH, Kim JS, Lee NJ. Concurrent chemoradiation followed by adjuvant chemotherapy in patients with locoregionally advanced nasopharyngeal carcinoma in Korea. Cancer Chemother Pharmacol. 2010; 66:643-51.

15. Lee AW, Tung SY, Chan AT, Chappell R, Fu YT, Lu TX, Tan T, Chua DT, O'Sullivan B, Tung R, Ng WT, Leung TW, Leung SF, et al. A randomized trial on addition of concurrent-adjuvant chemotherapy and/or accelerated fractionation for locally-advanced nasopharyngeal carcinoma. Radiother Oncol. 2011; 98:15-22.

16. Kwong DL, Sham JS, Au GK, Chua DT, Kwong PW, Cheng AC, Wu PM, Law MW, Kwok CC, Yau CC, Wan KY, Chan RT, Choy DD. Concurrent and adjuvant chemotherapy for nasopharyngeal carcinoma: a factorial study. J Clin Oncol. 2004; 22:2643-53.

17. Chen L, Hu CS, Chen XZ, Hu GQ, Cheng ZB, Sun Y, Li WX, Chen YY, Xie FY, Liang SB, Chen Y, Xu TT, $\mathrm{Li} \mathrm{B}$, et al. Concurrent chemoradiotherapy plus adjuvant chemotherapy versus concurrent chemoradiotherapy alone in patients with locoregionally advanced nasopharyngeal carcinoma: a phase 3 multicentre randomised controlled trial. Lancet Oncol. 2012; 13:163-71.
18. Yang S, Lin S, Fu Q, Cai B, Kong F, Huang G, Li F, Wang H. The effect of adjuvant chemotherapy on survival in patients with residual nasopharyngeal carcinoma after undergoing concurrent chemoradiotherapy. PLoS One. 2015; 10:e0120019.

19. Liang Z, Zhu X, Li L, Qu S, Liang X, Liang Z, Su F, Li Y, Zhao W. Concurrent chemoradiotherapy followed by adjuvant chemotherapy compared with concurrent chemoradiotherapy alone for the treatment of locally advanced nasopharyngeal carcinoma: a retrospective controlled study. Curr Oncol. 2014; 21:e408-17.

20. Chen Y, Sun Y, Liang SB, Zong JF, Li WF, Chen M, Chen L, Mao YP, Tang LL, Guo Y, Lin AH, Liu MZ, Ma J. Progress report of a randomized trial comparing long-term survival and late toxicity of concurrent chemoradiotherapy with adjuvant chemotherapy versus radiotherapy alone in patients with stage III to IVB nasopharyngeal carcinoma from endemic regions of China. Cancer. 2013; 119:2230 38.

21. Lee AW, Tung SY, Ngan RK, Chappell R, Chua DT, Lu TX, Siu L, Tan T, Chan LK, Ng WT, Leung TW, Fu YT, Au GK, et al. Factors contributing to the efficacy of concurrent-adjuvant chemotherapy for locoregionally advanced nasopharyngeal carcinoma: combined analyses of NPC-9901 and NPC-9902 Trials. Eur J Cancer. 2011; 47:656-66. 